\title{
Um aplicativo social para localização de doadores e receptores de sangue utilizando a plataforma OpenSocial
}

\author{
José Augusto Saraiva Lustosa Filho ${ }^{1}$ \\ Íthalo Bruno Grigório de Moura ${ }^{1}$ \\ João Phellipe de Freitas Pinto ${ }^{1}$ \\ Roberto Douglas Costa $^{1}$
}

\begin{abstract}
Resumo: As redes sociais têm ganhado destaque nos últimos anos em razão da facilidade de interação proporcionada aos seus usuários. Os aplicativos sociais tornaram-se um meio bastante atraente, expandindo as formas tradicionais de interação entre membros de uma rede social. O presente trabalho faz uma breve abordagem sobre o histórico da doação de sangue no Brasil. Mostra as tecnologias relacionadas ao OpenSocial, bem como a arquitetura da plataforma e dos aplicativos sociais. Por fim, apresenta as características e funcionalidades do protótipo desenvolvido, o qual possibilitará que membros de uma rede social possam localizar outros usuários ou grupos de usuários que estejam predispostos a doar um tipo sanguíneo específico.
\end{abstract}

Palavras-chave: Doação de sangue. OpenSocial. Redes sociais.

\begin{abstract}
Social networks have gained prominence in recent years due to the ease of interaction provided to its users. The social applications have become fairly attractive, expanding traditional forms of interaction between members of a social network. This paper takes a brief overview about the history of blood donation in Brazil. Shows the technologies related to OpenSocial and the platform architecture and social applications. Finally, it presents the features and functionality of the prototype. The prototype will enable members of a social network to find other users or groups of users who are predisposed to donate a specific blood type.
\end{abstract}

Keywords: Blood donation. OpenSocial. Social networks.

\section{Introdução}

A participação da população no processo de doação de sangue torna-se imprescindível para a manutenção dos níveis de estoque de bolsas sanguíneas nos hemocentros.

Atualmente, apesar de mais de um milhão de bolsas de sangue serem coletadas anualmente em todo mundo, estima-se que essa quantidade ainda não seja adequada para suprir a demanda global [1].

A Organização Mundial de Saúde recomenda que 3 a 5\% da população adulta seja doadora voluntária de sangue, a fim de atender a demanda transfusional média de cada país [2]. Em 2008 o Brasil alcançou a histórica marca de $2,2 \%$ de doações na população, sendo $1,8 \%$ realizadas na rede pública. No entanto, diante das disparidades regionais, como variações demográficas, disponibilidade de leitos de saúde e amplitude logística regional, esse percentual de doações também se mostra desigual em certas regiões, ora para cima ora para baixo [2].

A deficiência na captação e fidelização de doadores de sangue torna-se um problema crônico nos centros de coleta de sangue em todo país. Aliado à falta de informação acerca do processo de doação de sangue e à deficiência dos hemocentros na fidelização do doador, se fazem necessárias constantes campanhas de doação de sangue em todo país.

\footnotetext{
${ }^{1}$ Mestrado em Ciência da Computação, UERN - Rua Almino Afonso, 478 centro - Mossoró (RN) - Brasil

\{augusto.unix, ithalobgm, sr.phellipe, robertodcostalgmail.com\}
}

http://dx.doi.org/10.5335/rbca.2012.1814

Revista Brasileira de Computação Aplicada (ISSN 2176-6649), Passo Fundo, v. 4, n. 1, p. 12-24, mar. 201212 
Houve aumento expressivo de redes sociais e sites de relacionamento nos últimos anos. Todos proporcionando a interação entre pessoas conhecidas ou não. A rede social Facebook atualmente conta com mais de quinhentos milhões de usuários ativos e uma média de 50\% dos usuários entram na rede diariamente, consagrando-a como a mais popular do mundo. No Brasil, tem-se presenciado o sucesso de outra rede social, o Orkut.

Em 2007, o Facebook tornou público sua API (Application Programming Interface), também chamada "Facebook Plataform", para que desenvolvedores independentes pudessem criar aplicativos que interagissem com o usuário da rede social [4]. Qualquer pessoa com conhecimentos em programação e especificação da API do Facebook pode criar aplicativos que podem ser usados por qualquer usuário dessa rede. No entanto, esses aplicativos são restritos ao Facebook, não funcionando fora dele.

Com o sucesso desses aplicativos sociais, várias outras redes sociais demonstraram interesse em disponibilizar aplicativos aos seus usuários. Então, o Google, com apoio de várias empresas, criou o OpenSocial, uma plataforma que fornece APIs para que desenvolvedores independentes possam desenvolver aplicativos sociais para interagir com usuários de uma rede social.

A maior vantagem do OpenSocial, em relação a outras APIs, é a possibilidade de se desenvolver apenas um aplicativo para ser executado em diferentes redes, ou seja, a API do OpenSocial padroniza o aplicativo social, possibilitando sua execução com finalidades diversas.

Este trabalho é dividido em quatro seções. A seção 2 congrega o referencial teórico do trabalho, sendo abordados desde os aspectos históricos da doação de sangue até os motivos associados à intençãodo ato. Nas seções seguintes são feitas descrições sobre redes sociais e a plataforma OpenSocial, sendo abordadas as tecnologias associadas ao OpenSocial, os trabalhos relacionados e a própria arquitetura do aplicativo.

\section{Doação de sangue}

A participação da população na doação de sangue torna-se imprescindível para a manutenção satisfatória dos níveis de estoques de bolsas de sangue, evitando que a demanda seja superior à sua reposição. $\mathrm{O}$ processo de conquista e fidelização de doadores torna-se um fator chave para que centros de armazenamento mantenham seus estoques regulares.

As campanhas de doação, veículadas na mídia, buscam informar e conscientizar a população para a importância desse ato, assim como tentam introduzir esse hábito na cultura das pessoas.

Nos dias atuais, apesar de mais de um milhão de bolsas de sangue serem coletadas anualmente no mundo, estima-se que essa quantidade não seja suficiente para suprir a demanda global [1]. Em 2007, 162 países forneceram dados para a OMS que resultam no número de 85,4 milhões doações de sangue. Os dados provêm de países que respondem por um total de 5,9 bilhões de pessoas, representando $92 \%$ da população mundial. O relatório abrange 7997 centros de sangue, que coletaram, em média, 9000 doações por centro. Nos países desenvolvidos, a taxa anual média de recolha por centro foi de 13.600; nos países em transição, 6.000; e nos países em desenvolvimento 2.800 [5].

\subsection{Aspectos históricos}

O homem sempre teve fascínio pelo sangue, fato que pode ser comprovado pelos ritos e sacrifícios promovidos pelos povos da Antiguidade e pelo costume de dar sangue para os doentes, na cultura greco-romana [6]. O sangue, pelos povos antigos, era considerado símbolo de força e vitalidade, que levava o homem à infusão em suas veias com a intenção de vencer a idade e as doenças [3].

Em Oxford, no ano de 1665, foram realizadas as primeiras transfusões em animais. Dois anos depois, Jean Baptiste Denis, professor de matemática e filosofia na cidade de Montipellier, por meio de um tubo de prata, infundiu um copo de sangue de carneiro em Antoine Mauroy, de 34 anos, doente mental, que veio a falecer após a terceira transfusão. Na época, as transfusões eram heterólogas e o professor defendia sua prática argumentando que sangue de animais continha menos vícios e paixões [7].

Ainda no século XIX, muitos problemas com coagulação de sangue e reações adversas continuavam a aparecer e desafiar os cientistas [7]. Em 1818, James Blundell realizou, com êxito, a primeira transfusão com sangue humano, em mulheres com hemorragias pós-parto [7].

Revista Brasileira de Computação Aplicada (ISSN 2176-6649), Passo Fundo, v. 4, n. 1, p. 12-24, mar. 201213 
Karl Landsteiner marcou o fim da fase empírica e o início da científica ao descrever o grupo sanguíneo ABO, em 1900, servindo de base científica para a compreensão das compatibilidades entre indivíduos [2]. Vários fatos ocorridos na primeira metade do século XX favoreceram a utilização do sangue em terapias, como o uso de citrato de sódio como anticoagulante e o resfriamento do sangue, que permitiram o início do processo de estocagem, em 1930, nos centros de hematologia e transfusão de sangue instalados por todo mundo [6].

No final da primeira metade do século XX, Landsteiner descobriu o Fator Rh, trazendo uma base sólida para a compatibilidade das transfusões sanguíneas e seus componentes [2].

Na década de 1950 foi introduzida a bolsa plástica em substituição aos frascos de vidro, melhorando significativamente as condições de armazenamento, conservação e transporte do sangue. Na mesma época, teve início a utilização de centrífuga refrigerada, possibilitando a consolidação da terapia por componentes. Também durante a segunda metade do século XX, surgiram novas técnicas cirúrgicas e de tratamentos, aumentando a demanda por sangue [8].

No início da década de 1980, decorrente da epidemia de aids e da contaminação transfusional pelo HIV, ocorreu uma grande conscientização e preocupação em diminuir os riscos transfusionais, intensificando o uso e desenvolvimento de técnicas de triagem laboratoriais [8]. Nesse período, a adoção de políticas com maior rigor no processo de doação de sangue trouxe um decréscimo no número de indivíduos aptos à doação de sangue [9].

\subsection{Doação de sangue no Brasil}

Em 1969, a Organização Mundial da Saúde (OMS) realizou um diagnóstico do sistema transfusional brasileiro, caracterizando-o pela falta de ações ordenadas e normatizadas e precárias atividades fiscalizadoras. O atendimento ao público era feito com pouca participação do sistema público, além de outros serviços serem executados pela iniciativa privada. A comercialização de sangue e a utilização intensa de doadores remunerados completavam o quadro da hemoterapia brasileira [2].

A Sociedade Brasileira de Hematologia e Hemoterapia (SBHH) promoveu, em 1979, campanhas visando ao estímulo da doação voluntária de sangue em conjunto com a política reguladora que definia a doação de sangue exclusivamente voluntária (BRASIL, 2011). Para a imediata reposição dos níveis de estoque, os serviços de hemoterapia possibilitaram a doação de familiares para pacientes hospitalizados, os chamados "doadores de reposição" [10].

Em 1980, o Ministério da Saúde cria o Programa Nacional do Sangue, Pró-Sangue, promovendo um avanço definitivo no processo de institucionalização profissional da área do sangue e hemoderivados. Dentre seus vários objetivos, tinha como foco estimular a doação voluntária, não remunerada e universal, instalando uma rede de centros de hematologia e hemoterapia, responsáveis por organizar os programas em cada estado [2].

$\mathrm{Na}$ mesma década, com o aparecimento da aids e a pressão social relativa aos riscos envolvidos durante as transfusões, é realizada a $8^{\text {a }}$ Conferência Nacional de Saúde em 1986, fomentando o debate sobre a saúde na futura Constituinte [2]. Em 1988, a Constituição Federal vetou qualquer tipo ou forma de comercialização de sangue e hemocomponentes, considerando o sangue uma questão estratégica e política, além de apoiar a hemorrede pública (BRASIL, 1988). No mesmo ano é sancionada uma lei federal que obriga o cadastramento de doadores e a realização de exames laboratoriais. Em 1989 foi publicada uma portaria aprovando normas técnicas destinadas a disciplinar os processos de coleta, processamento de transfusão, componentes e derivados [2].

No período de 1993 a 1996 foram publicadas portarias que determinavam a obrigatoriedade da realização de testes sorológicos, implantação de programas de controle de qualidade em sorologia, imunohematologia e de insumos utilizados em hemoterapia. Também houve acelerado crescimento da hemorrede pública e implementação de atividades de hemoterapia sanitária, através do Programa Nacional de Inspeções nas Unidades de Hemoterapia [8].

Em 1998 é instituída a Meta Mobilizadora Nacional do Ministério da Saúde, na qual preconizava "sangue com garantia de qualidade em todo seu processo até o ano de 2003", impulsionando o Programa Nacional do Sangue. Com a participação de técnicos dos estados, foram instituídos grupos de trabalho para implementação de 12 programas prioritários a serem desenvolvidos, destacando-se o Programa Nacional de Doação Voluntária de Sangue (PNDVS) [2]. 
Ainda que os programas estaduais de sangue tenham sido implementados gradativamente e não de forma homogênea nos estados da federação, trouxeram avanços significativos na melhoria da qualidade de coleta, armazenagem e distribuição do sangue coletado no país.

\subsection{Fatores motivacionais para doar sangue}

Um estudo realizado na Espanha, em 1996, destacou o altruísmo, a pressão social e a recompensa entre os fatores que motivaram a doação de sangue [14]. Em outro estudo, realizado no Brasil no Hemocentro de Belo Horizonte/Hemominas, verificou-se a existência de tabus e crenças entre os candidatos à doação de sangue, tais como a hipótese de que doar sangue provoca o aumento ou redução da pressão arterial. Constatou-se que havia falta de esclarecimento acerca do processo de doação sanguínea, imperando velhos mitos sobre o processo de doação de sangue em uma parcela dos candidatos [11].

$\mathrm{Na}$ Espanha, a análise do discurso de cinco grupos de doadores regulares de sangue mostrou que o processo é compreendido racionalmente. Porém, o mesmo é avaliado de maneira emocional, personalizada e estereotipada pelos indivíduos [12].

Fatores socioeconômicos poderiam estar entre as razões que dificultam a doação de sangue, como a pouca integração em atividades comunitárias e a alta competitividade no mercado de trabalho. Além desses fatores, o envelhecimento populacional e uma política rígida para a obtenção de um maior nível de segurança transfusional podem distanciar indivíduos predispostos a doar sangue [9].

A deficiência na captação e fidelização de doadores torna-se um problema crônico nos centros de coleta de sangue em todo país. Aliado à falta de informação acerca do processo de doação e a deficiência dos hemocentros na fidelização do doador, fazem-se necessárias constantes campanhas de doação de sangue em todo país.

\section{Redes sociais}

As redes sociais podem ser caracterizadas como um conjunto de participantes autônomos, concentrando ideias e recursos em torno de valores e interesses compartilhados [13]. Uma rede social também pode ser compreendida como um conjunto de indivíduos ligados entre si por um conjunto de relações [15].

A teoria de redes sociais caracteriza os relacionamentos sociais aos nós e conexões entre estes. O nó representa um ator na rede social e as conexões refletem alguma forma de ligação entre os atores. Existem várias formas de conexão entre os atores, cada uma representando contextos distintos acerca da rede social [16].

A disseminação de informação em grupos é bastante comum em redes sociais, em razão de os atores aproximarem-se de outros que lhe inspirem confiança ou que possuam relações de amizade, e em muitos casos relações profissionais também. Dessa forma, as redes sociais vão crescendo à medida que os contatos vão sendo feitos, resultando na construção social dos indivíduos, cujas similaridades formam um corpo social, cujas unidades são as redes sociais. As relações mantidas na rede social são tão imbricadas que, na maioria das vezes, é difícil precisar como começaram ou com quem começou [17].

O Orkut é uma das redes sociais mais populares no Brasil, é mantida pela Google, que funciona basicamente através de perfis e comunidades. Os perfis são criados pelas pessoas ao efetuarem o cadastro, assim como uma sugestão de amigos é oferecida aos usuários iniciais. As comunidades formadas pelos perfis são criadas pelos próprios usuários, que agregam vários outros perfis de interesse comum, funcionando como fóruns, nos quais opiniões sobre determinado assunto podem ser expressas.

A rede social Orkut proporciona duas formas de intereção social mediada por computador, a interação mútua [20] e a interação reativa [20]. A interação mútua [20] pode ser caracterizada pelas mensagens inseridas nas comunidades quando qualquer usuário escreve e recebe mensagens de respostas na comunidade e também quando usuários trocam mensagens univocamente entre si. A interação reativa [20] pode ser observada quando um usuário solicita amizade a outro, assim como também pode ser verificada quando um usuário classifica seus amigos conforme sua afinidade.

Outra forma de interação entre os membros do Orkut foi introduzida pela adoção da plataforma OpenSocial, a qual permitia execução de aplicativos sociais para uso dos membros do Orkut. Um aplicativo social poderia vir a estimular e intermediar interações entre os usuários da rede social.

Revista Brasileira de Computação Aplicada (ISSN 2176-6649), Passo Fundo, v. 4, n. 1, p. 12-24, mar. 201215 


\section{Plataforma OpenSocial}

O OpenSocial é uma plataforma de desenvolvimento de aplicativos sociais que possibilita a implantação do aplicativo social em várias redes sociais. É a única plataforma que foi criada com objetivo de permitir seu uso em várias redes sociais, além de ser uma biblioteca de código aberto para desenvolvedores de aplicações. A plataforma foi concebida para facilitar a implantação de um único aplicativo em várias redes sociais [18].

O código de amostra do OpenSocial é fornecido sob a licença Apache 2.0 e sua documentação licenciada por meio da Creative Commons [5].

Quase nenhuma rede social permitia desenvolvedores independentes desenvolverem seus próprios aplicativos para as redes sociais. A possibilidade de fato surgiu com a API do Facebook. O OpenSocial foi criado para permitir que desenvolvedores independentes pudessem criar aplicativos que fossem implantados em várias redes sociais, ao contrário da API do Facebook [18].

Atualmente, os aplicativos sociais não são destinados apenas para serem populares (virais), mas que, além disso, retenham usuários por um longo prazo, com interações mais complexas e por mais tempo de uso. Aplicações envolvendo músicas, mídias e educação são exemplos desse modelo [18].

Com o lançamento do OpenSocial, desenvolvedores independentes finalmente tinham um framework para desenvolver aplicativos sociais que seriam executados em qualquer site que implementasse a especificação do OpenSocial. A plataforma OpenSocial foi rapidamente adotada por muitas redes sociais tradicionais, tais como MySpace, Orkut, Hi5 e Ning. O benefício para as redes sociais que adotaram o OpenSocial foi imediato, pois já tinham uma considerável quantidade de desenvolvedores do OpenSocial que implementavam aplicativos sociais. Dessa forma, os desenvolvedores se beneficiaram, pois poderiam rapidamente implementar uma aplicação portável a várias redes sociais, não ficando restrita apenas a uma rede social [5].

No Quadro 1 são apresentadas as redes sociais que implementam a especificação do OpenSocial e suas versões.

\begin{tabular}{|lc|}
\hline Rede Social & Versão do Open Social \\
\hline hi5 & 0.8 \\
Linkedln & 0.8 \\
Netlog & 0.8 \\
MySpace & 0.8 \\
Webon & 0.8 \\
XiaoNet & 0.8 \\
Yahoo! & 0.8 \\
51.com & 0.7 \\
FanBox & 0.7 \\
Freebar & 0.7 \\
Friendster & 0.7 \\
Hyves & 0.7 \\
IDtall & 0.7 \\
Maill.ru & 0.7 \\
Ning & 0.7 \\
orkut & 0.7 \\
YiOt & 0.7 \\
lokalisten & 0.8 \\
IGoogle & 0.8 \\
imeem & 0.7 \\
Viadeo & 0.7 \\
CrylN & 0.7 \\
Tlanya & 0.7 \\
Plaxo Pulse & 0.5 \\
\hline
\end{tabular}

Quadro 1: Redes sociais e versão de suas especificações. Adaptado de Grewe, 2009.

\footnotetext{
${ }^{3}$ Fator Rh é um dos dois grupos de antígenos eritrocitários de maior importância clínica, estando envolvido nas reações transfusionais hemolíticas.

Os aplicativos sociais possuem três diferentes interfaces: a visão "canvas", que apresenta a "tela cheia" do aplicativo no container da rede social; a visão "home", quando o aplicativo está sendo executado na página inicial, assim que o usuário entra na rede social; e a visão "profile", que é exibida quando o aplicativo está sendo executado na página do perfil do usuário [18].
} 


\subsection{Arquitetura do OpenSocial}

A arquitetura de um aplicativo implementado para funcionar no OpenSocial envolve um cliente (geralmente um navegador web) e um recipiente(geralmente uma rede social). Qualquer requisição feita pelo cliente a um aplicativo social será intermediado pelo recipiente, que pode inclusive "filtrar" os resultados antes que sejam entregues ao cliente. Mesmo que o aplicativo social seja hospedado em servidores externos à rede social, pode ser necessária a autenticação que em alguns casos pode ser feita pelo protocolo OAuth [18].

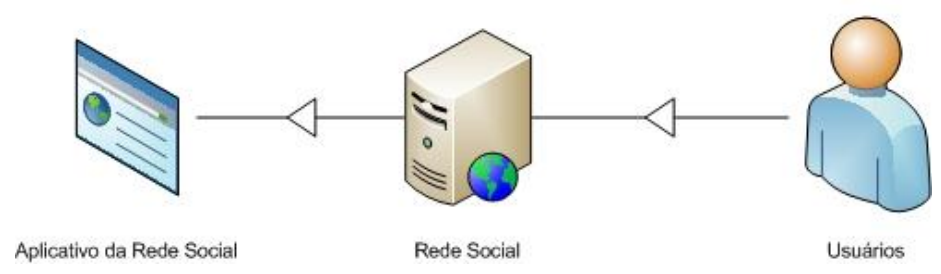

Figura 1: Arquitetura entre a rede social e o aplicativo social

A Figura 1 exemplifica a arquitetura imposta entre a rede social e o aplicativo social. Quando o cliente realiza chamadas para o código hospedado externamente à rede social, esta faz chamadas à aplicação social e devolve os resultados ao cliente (navegador do usuário).

\subsection{Arquitetura de um aplicativo social}

Um aplicativo baseado na API JavaScript do OpenSocial pode ser visto como um objeto em camadas, como exemplifica a Figura 2. Uma camada externa especifica o aplicativo como um gadget Google usando XML. O conteúdo interno ao XML é o conteúdo do aplicativo que consiste tipicamente de HTML e por vezes CSS (Cascading Style Sheet) e outro conteúdo.

Os aplicativos sociais que fazem uso da API cliente utilizam a API JavaScript do OpenSocial para fazer consultas ao recipiente de forma assíncrona para a maioria das chamadas. A maior parte dos métodos do OpenSocial não retorna os dados diretamente, ou seja, o aplicativo social deve requisitar os dados, e então, algum tempo depois, o recipiente retornará os resultados para a função callback no aplicativo [18]. A Figura 2 ilustra a comunicação assíncrona entre o gadget (aplicativo do OpenSocial) e o recipiente (rede social).

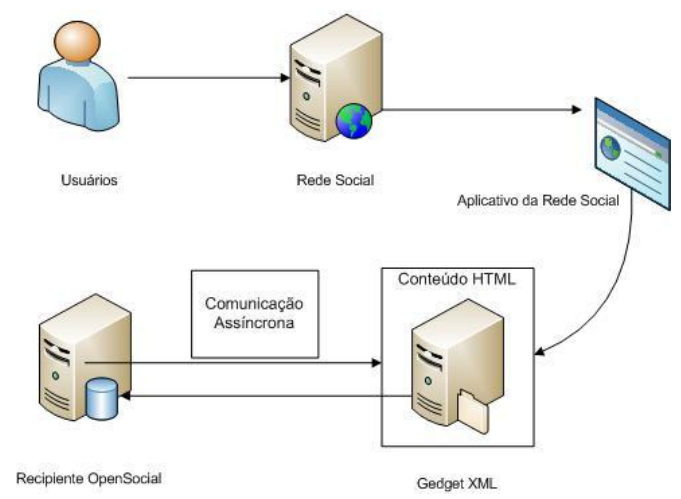

Figura 2: Camadas de um aplicativo OpenSocial e comunicações assíncronas com o recipiente.

Um aplicativo social pode fazer vários pedidos durante uma sessão com um usuário, mas também pode optar por pedidos de lote de uma só vez (para melhorar o desempenho). Por exemplo, um pedido para obter o nome do usuário ou uma lista de amigos de um usuário, a aplicação envia uma solicitação usando o método de 
envio send, da classe DataRequest. Depois que o recipiente recebe um DataRequest, ele processa a requisição a fim de devolver as informações solicitadas [18].

\section{Trabalhos relacionados}

Em razão da crescente utilização das redes sociais é cada vez mais comum a criação de aplicativos e tecnologias para a promoção de algum benefício para os que utilizam esse mecanismo de interatividade. Atualmente muitos trabalhos estão sendo desenvolvidos para utilizar o potencial que as redes sociais oferecem. Seguindo essa proposta, as redes sociais têm contribuído para o desenvolvimento da e-Health, que oferece características promissoras de serviços de saúde a qualquer hora e em qualquer lugar.

No trabalho realizado por Rahmarnet al.[21] é proposto um framework, chamado "SenseFace", que pode passar dinamicamente dados sensoriais a partir de uma rede de sensores corporais à sua rede social e viceversa. Uma rede de sensores corporais (BSN) pode capturar fenômenos físicos a partir de informações fornecidas pelo corpo humano, contextos ambientais e eventos de alto nível de uma pessoa. Tal processo inclui receber os dados sensoriais, analisando-o para acionar os serviços necessários, tais como o envio de uma mensagem de alerta para o médico de família, hospital, serviço de emergência, membros da família, amigos, e assim por diante. Além disso, uma BSN também permite que os membros de uma rede social consultem em tempo real dados contextuais e eventos. Com isso, a combinação de uma rede social com uma BSN melhora o atual estado da arte em aplicações de $e$-health.

\section{Protótipo}

Tendo feito um estudo das APIs do OpenSocial, este capítulo apresenta a análise e a implementação do aplicativo social. Foram utilizadas as linguagens HTML e Javascript para a implementação do protótipo, assim como se fez uso do ambiente de desenvolvimento Eclipse associado ao Plug-in OSDE. Este possibilitou uma abstração do ambiente real, uma rede social, por meio de seus vários componentes.

\subsection{Descrição}

A análise do SocialBlood utilizou diagramas da linguagem de modelagem UML (Unifield Modeling Language). As figuras a seguir ilustram alguns casos de uso e diagramas de atividades do protótipo.

\subsubsection{Casos de uso}

A Figura 3 ilustra um diagrama com os principais casos de uso do protótipo e a descrição de cada caso de uso.

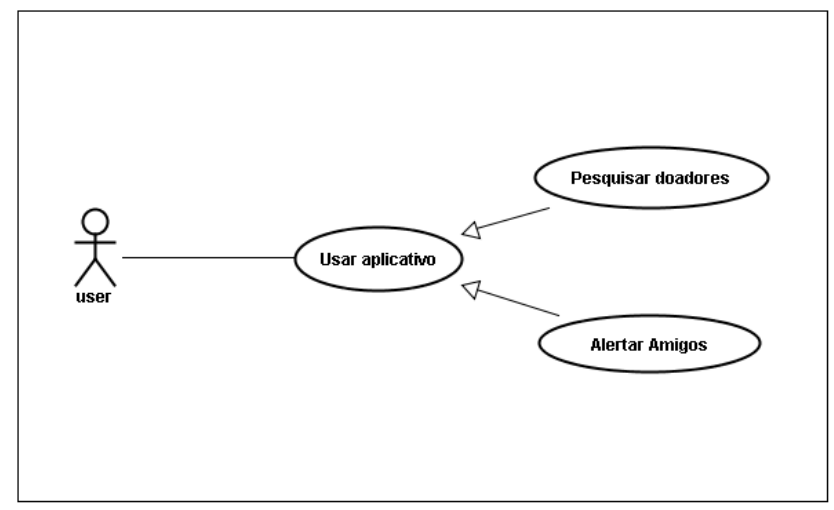

Figura 3: Diagrama de casos de uso 
Caso de uso: usar aplicativo.

Descrição: corresponde ao acesso ao aplicativo por meio da rede social.

Precondição: o usuário deve ter adicionado o aplicativo social ao seu perfil.

Pós-condição: ao final da utilização do aplicativo social, ao usuário deve ter sido permitido buscar amigos predispostos a doar sangue, conforme o tipo sanguíneo selecionado, e alertar os amigos do usuário tanto pela sua disponibilidade em doar sangue quanto pela sua necessidade por um tipo sanguíneo específico por meio de atualizações do usuário.

\section{Fluxo de eventos:}

1. o usuário entra na rede social através de uma conta criada previamente.

2. deve-se adicionar o aplicativo social ao perfil utilizado.

3. atualizar dados pessoais no aplicativo social.

Caso de uso: pesquisar doadores

Descrição: corresponde ao processo de buscar dentre os amigos do usuário aqueles que contêm o tipo sanguíneo requisitado e que estejam predispostos a doar sangue.

Precondição: o usuário deve ter adicionado o aplicativo social ao seu perfil na rede social, além de ter amigos adicionados ao seu perfil que utilizem o aplicativo.

Pós-condição: ao final da pesquisa de doadores, o usuário deve obter uma lista de amigos com o tipo sanguíneo correspondente ao selecionado na pesquisa, além do que estes devem ser indivíduos predispostos a doar sangue.

\section{Fluxo de eventos:}

1. informar o grupo sanguíneo desejado.

2. exibir usuários com tipo sanguíneo pesquisado e predispostos a doar sangue.

Caso de uso: alertar amigos.

Descrição: corresponde ao processo de alertar os amigos do usuário na rede social que está apto a doar sangue, ou alertar que está precisando de um determinado tipo sanguíneo.

Precondição: o usuário deve ter adicionado o aplicativo social ao seu perfil, ter atualizado os dados pessoais no aplicativo social e amigos na rede social, sendo que estes não necessariamente devem ter adicionado o aplicativo ao seu perfil.

Pós-condição: ao final do caso de uso Alertar Amigos, ao usuário será permitido gerar alertas, na forma de atualizações que são visíveis a todos os amigos do mesmo, indicando sua disponibilidade para doar sangue ou uma necessidade por um determinado tipo sanguíneo.

\section{Fluxo de eventos:}


1. o usuário tem a opção de alertar os amigos, gerando um alerta a todos os amigos do usuário, independentemente se os mesmos estejam ou não utilizando o aplicativo social;

2. caso o usuário tenha selecionado a opção de gerar um alerta para a necessidade de um determinado sangue, o aplicativo na forma de uma atualização na rede social informa aos amigos do usuário da necessidade do tipo sanguíneo escolhido.

\subsubsection{Diagrama de atividades}

\section{- Pesquisar doadores}

A Figura 4 ilustra o diagrama de atividades que representa o fluxo do caso de uso pesquisar doadores.

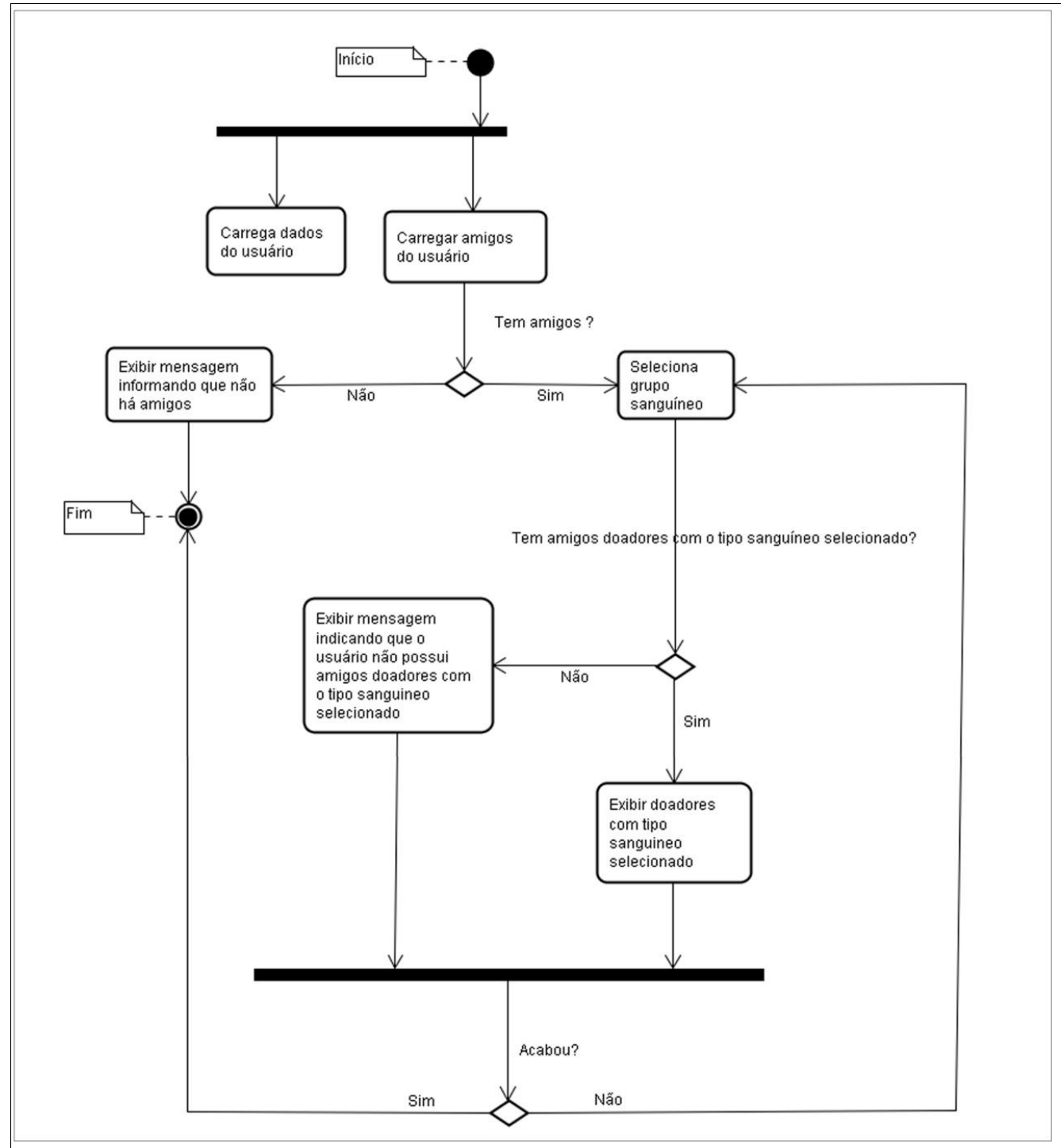

Figura 4: Diagrama de atividades Pesquisar doadores

\section{- Alertar amigos}


Na Figura 5 é exibido o diagrama de atividades que representa o fluxo do caso de uso Alertar amigos.

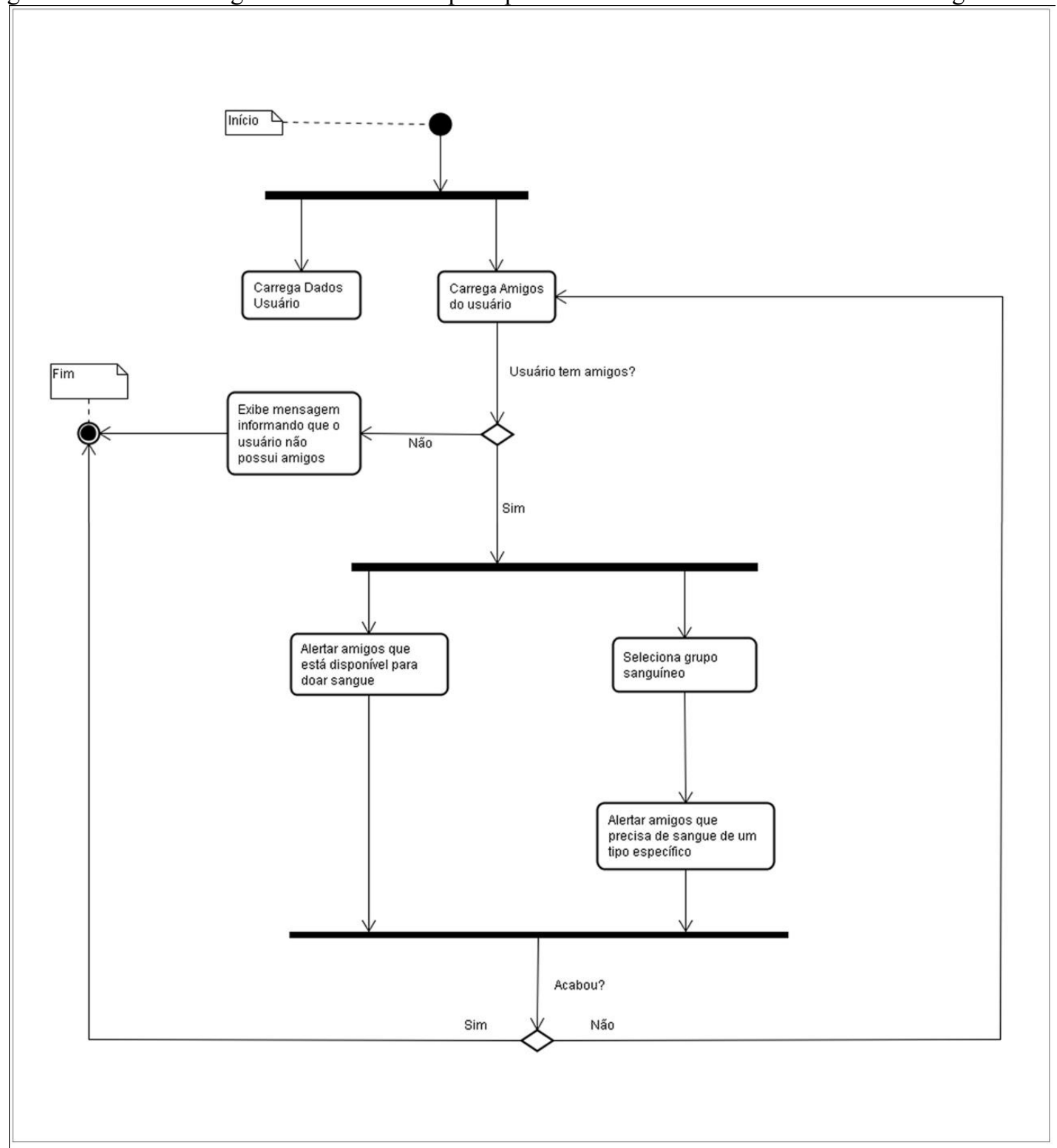

Figura 5: Diagrama de atividades Alertar amigos

\subsection{Descrição funcional}

Através do diagrama de telas visualizado na Figura 6, é possível observar todas as funcionalidades oferecidas pelo protótipo SocialBlood. Todas essas funcionalidades são oferecidas por meio de uma rede social que implementa as especificações do OpenSocial. 


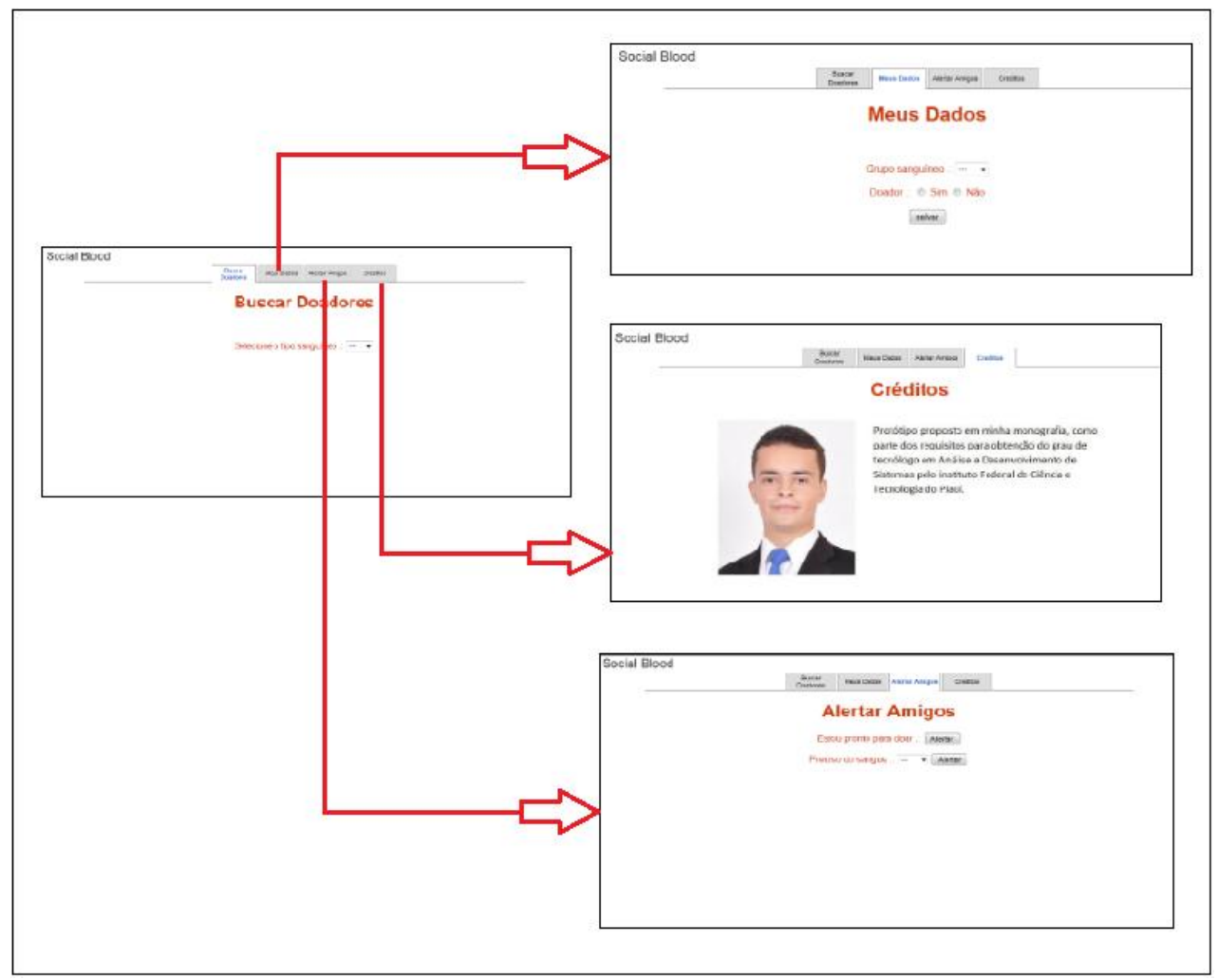

Figura 6: Diagrama de telas do protótipo

Como mostra a Figura 6, a tela inicial da aplicação tem um menu com quatro opções: Buscar doadores, Meus dados, Alertar amigos e Créditos. Na primeira opção, Buscar doadores, o usuário pode buscar entre seus amigos na rede social que possuem o SocialBlood associado ao seu perfil, aqueles que possuem determinado tipo sanguíneo e que estejam pré-dispostos a doar sangue, ou seja, a funcionalidade Buscar doadores possibilita o encontro de indivíduos ou grupos que tenham em algum momento manifestado a predisposição em realizar a doação de sangue.

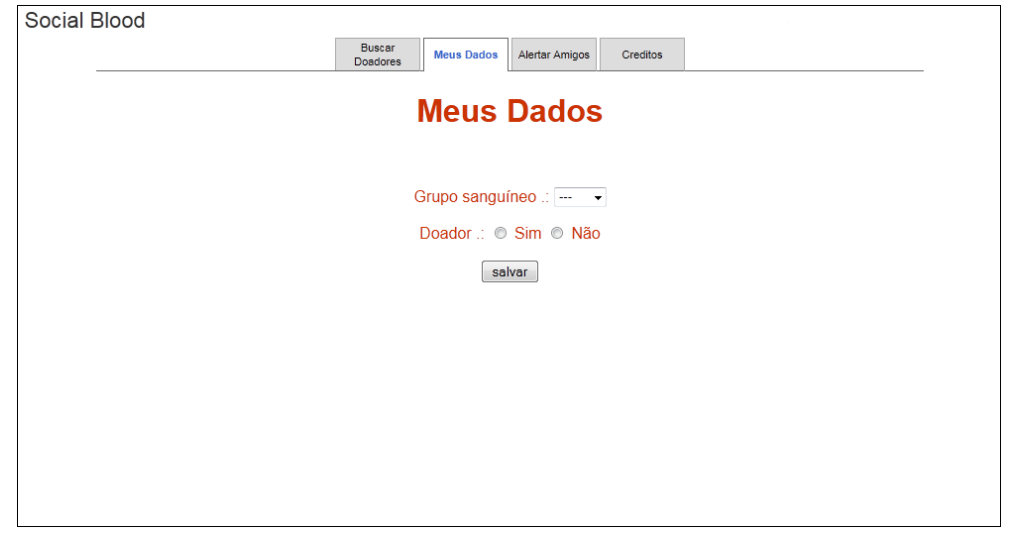

Figura 7: Tela de atualização dos dados pessoais

A opção de menu Meus dados, ilustrado na Figura 7, possibilita a atualização de dados do usuário, tais como o tipo sanguíneo e a intenção de uso do aplicativo social exemplificado no campo Doador.

A terceira opção de menu, Alertar amigos, ilustrado na Figura 8, possibilita ao usuário gerar alertas na forma de atualizações, visíveis a todos seus amigos da rede social, independentemente se os amigos do usuário possuem ou não, associados às suas contas, o aplicativo SocialBlood. A primeira opção para gerar o alerta 
indica que o usuário corrente está disponível e predisposto a realizar a doação. A segunda opção gera uma mensagem, conforme o tipo sanguíneo selecionado, indicando a necessidade de um tipo sanguíneo específico.

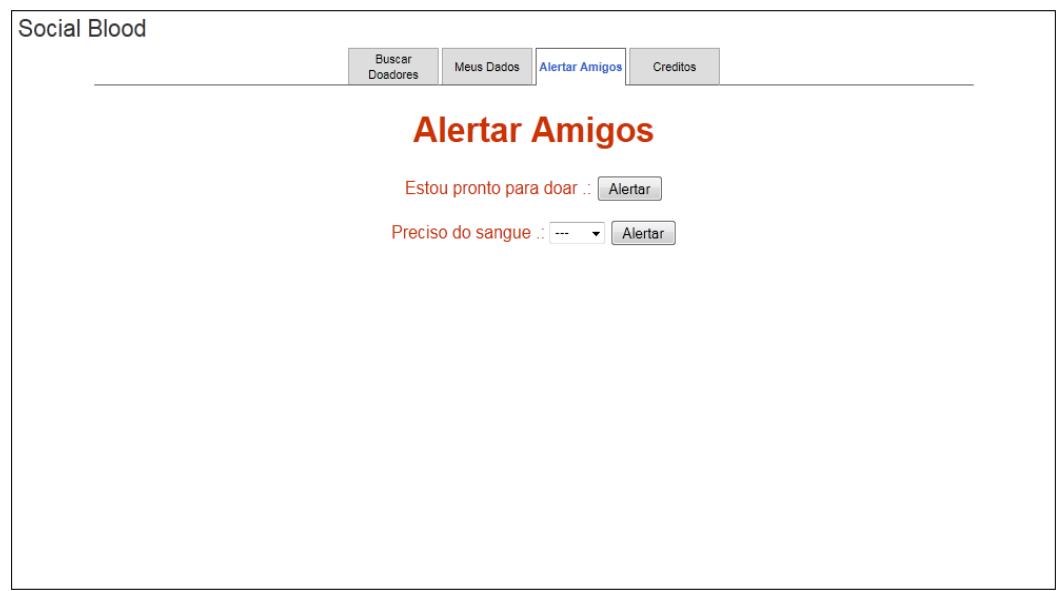

Figura 8: Tela da funcionalidade Alertar amigos

\section{Conclusão}

Por meio deste trabalho foi possível realizar um estudo sobre a plataforma OpenSocial e também sobre algumas tecnologias associadas para o desenvolvimento de aplicativos para redes sociais, observando suas principais características. Possibilitando elicitar as vantagens do OpenSocial frente a outras plataformas de desenvolvimento de aplicativos sociais.

Com o estudo da plataforma OpenSocial, notou-se que a mesma disponibiliza muitas funcionalidades interessantes, tais como acesso aos dados pessoais dos usuários de uma rede social e a possibilidade de promover interação entre os membros da rede social, que foi a funcionalidade mais importante.

Esse protótipo traz como principal vantagem a possibilidade de pessoas que estejam necessitando de uma transfusão sanguínea possam encontrar, por meio de uma rede social, grupos ou usuários que estejam predispostos a doar sangue.

Apesar de vários fatores influenciarem na aceitação do protótipo por parte dos usuários de uma rede social, acredita-se que este irá auxiliar e agilizar o processo de localização de indivíduos predispostos a doar sangue, fazendo com que seja poupada, pelo menos, uma unidade de sangue que seria retirada de algum hemocentro.

\section{Referências}

[1] BUCIUNIENE, I; et al. Blood donors' motivation and attitude to non remunerated blood donation in Lithuania. 2BMC Public Health, 2006.

[2] BRASIL. Ministério da Saúde. Coordenação de Sangue e Hemoderivados. In: ENCONTRO NACIONAL DE PROFISSIONAIS NA ÁREA DE RECRUTAMENTO DE DOADORES DE SANGUE, II. Brasília, 1991.

[3] ALMEIDA, M. B. Uma introdução ao XML, sua utilização na internet e alguns conceitos complementares. Belo Horizonte, 2002.

[4] KIRKPATRICK, David. Facebook's plan to hook up the world. Disponível em: $<$ http://money.cnn.com/2007/05/24/technology/facebook.fortune/>. Acesso em: 4 abr. 2011.

[5] WHO. Word Health Organization. Disponível em: http://www.who.int/. Acesso em: 22 abr. 2011. 
[6] RIZZI, M. História de la transfusion de sangre. Sus comienzos em Uruguay. Rer. Méd. Uruguay, n. 15, p. 165-182, 1999.

[7] GINGERICH, D. A. Fluid, shock and blood therapy. In: HOWARD, J. L. Current veterinary therapy. Food animal practice 2. Philadelphia: W.B. Saunders Company, 1986. p. 1-8.

[8] SERINOLLI, M. I. Evolução da medicina transfusional no Brasil e no mundo. Rev. Hemat. Hemot. Fundação Pró-Sangue, Hemocentro de São Paulo, 1999.

[9] BEIJAR, J. E. The donor/demand dilemma. Trans. Med. Update. Boletín "on-line", 1996. Institute for Transfusion Medicine Pittsburgh, Pennsylvania.

[10] GONÇALEZ T.; SABINO E. C.; CHAMONE D. F. Trends in the profile of blood donnors at a large blood center in the city of São Paulo, Brazil. Rev. Panam. Salud Pública, Edição especial, n. 13, v. 2/3, p. 114-148, 2003.

[11] RODRIGUES R. N.; et al. Aspectos sócio-demográficos e culturais da doação de sangue em Belo Horizonte. In: SEMINÁRIO SOBRE A ECONOMIA MINEIRA, VII. Anais... Diamantina, 1995. V. II, p.513531 .

[12] SUÁREZ, I. M. B.; et al. Blood donors and blood collection. How regular blood donors explain their behavior. Transfusion, n. 144, v. 10, p. 1441-1447, 2004.

[13] MARTELETO, R. M. Análise de redes sociais - aplicação nos estudos de transferência da informação. Brasília: Ci. Inf, 2001.

[14] FERNANDEZ M. A.; et al. Attitudes, beliefs, and motivations in blood donors and non-donors. Sangre (Barc), 1996.

[15] DOWNES, S. Semantic networks and social Networks. National Research Council Canada. The Learning Organization. v. 12, n. 5, 2005.

[16] WASSERMAN, S.; FAUST, K.; IACOBUCCI, D. Social network analysis: methods and applications. Cambridge University Press, 1994.

[17] TOMAÉL, M. I. Redes sociais, conhecimento e inovação localizada. Inf. Londrina, v. 12, n. esp., 2007.

[18] GREWE, L. OpenSocial Network Programming. Indianapolis: Wrox, 2009.

[19] OPENSOCIAL Containers. Disponível em:

$<$ http://wiki.OpenSocial.org/index.php?title=Containers\&oldid=4368>. Acesso em: 28 mar. 2011.

[20] PRIMO, Alex. Interação mútua e interação reativa: uma proposta de estudo. Disponível em: $<$ http://usr.psico.ufrgs.br/ aprimo/pb/intera.htm>. Acesso em: 06 jan. 2011.

[21] Rahman, M. A.; et al. A Framework to bridge social network and body sensor network: an e-Health perspective. In: INTERNATIONAL CONFERENCE ON MULTIMEDIA AND EXPO, (ICIME). Canadá, p. 1724-1727, 2009. 